\title{
Influence of Geometric Equations in Mixed Problem of Porous Micromorphic Bodies with Microtemperature
}

\author{
Lavinia Codarcea-Munteanu ${ }^{\dagger}$ and Marin Marin ${ }^{*}+$ ( $\mathbb{C}$ \\ Department of Mathematics and Computer Science, Transilvania University of Brasov, 500036 Brasov, Romania; \\ codarcealavinia@unitbv.ro \\ * Correspondence: m.marin@unitbv.ro \\ + These authors contributed equally to this work.
}

Received: 27 July 2020; Accepted: 17 August 2020; Published: 18 August 2020

\begin{abstract}
The study of the mixed initial-boundary value problem, corresponding to the thermoelasticity of porous micromorphic materials under the influence of microtemperatures, represents the main objective of this article. Achieving qualitative results on the existence, uniqueness and continuous dependence on the initial data and loads, of the solution of the mixed problem, implies a new perspective of approaching these topics, imposed by the large number of unknowns, which increases the complexity of equations and conditions that characterize the thermoelastic porous micromorphic materials with microtemperatures. The use of the semigroup theory of operators represents the optimal solution for deducing these results, the theory being adaptable to the requirements of the demonstrations, the mixed problem turning into a problem of Cauchy type, with regards to an equation of evolution on a Hilbert space, chosen appropriately.
\end{abstract}

Keywords: micromorphic; porous; thermoelasticity; microtemperature; existence; uniqueness; continuous dependence

\section{Introduction}

Aiming to remove the inadequacies between the theory and the results of its implementations, occurred, for example, in the case of granular bodies with large particles, where the effect of materials microstructure makes its presence felt, the theory of microstructure is optimally appropriate for applications in solid mechanics.

Part of the microstructure theory, with its bases founded by the Cosserat brothers, see [1], later approached by many other researchers, such as Green and Rivlin in [2], Mindlin in [3], the micromorphic theory was introduced by Eringen, see [4,5], to explore materials with microelements that deform separately from their motions.

In this theory, each particle is deformable, having twelve freedom degrees, six for the particle microdeformations, three for the microrotations and three for the particle motion.

Compared to the classical continuous mechanics, where material bodies are considered to be continuous assemblies of infinitesimal size material particles and not endowed with inner structure, the micromorphic theory regards the material bodies as a set of finite size particles having an inner structure.

The theory of micromorphic materials, with the diversity of exploration directions it offers, has been the subject of many research papers, some examples being highlighted below.

Eringen presented in [6] a new perspective on the micromorphic mechanics balance laws, studied in [7] the effect of the memory on the microelastic solids and microviscous fluids and developed the linear theory of the micromorphic thermoelastic solids in [8], by giving the linear constitutive 
equations, by formulating the initial-boundary value problems for anisotropic and isotropic cases and by revealing the relation between the micropolar and microstretch cases with the micromorphic ones.

The generalization and deepening of the micromorphic theory, regarding the micromorphic mixtures, can be found in $[9,10]$, where a detailed description of the behavior of certain materials, such as polycrystalline mixture, polymolecular crystal lattices or granular composites, has been created, both the balance equations and the general form of the constitutive equations being determined.

Several aspects of the micromorphic materials theory have been the subject of a wide number of papers, such as: [11-20].

The theory of porous materials, initially studied by Goodman and Cowin in [21], was subsequently deepened [22-24], being just a few examples of recent papers. First studies dedicated to the theory of bodies having microtemperatures were published by Grot, which created the foundation of this theory see, for instance [25], considering that the microelements of a continuous body are endowed with microtemperatures, along with microdeformations, thus extending existing theories of materials with inner structure.

In this context, the second law of thermodynamics, see [26], is modified in order to include the microtemperatures, and the equations corresponding to the first moment of energy are added to the classical law of equilibrium, related to a material with microstructure, these equations leading to the equations of microtemperatures thermal conductivity.

We must outline the great number of works in the theory of elasticity or thermoelaticity of bodies having microtemperatures, associated with various materials, some examples, in this regard, being [27-37].

Our present article approaches the effects of microtemperatures on the fundamental features of the mixed problem with initial and boundary values corresponding to the thermoelasticity theory of the micromorphic porous materials. In this context, our problem will be transformed into a problem of Cauchy type, attached to an evolutionary equation on a particular Hilbert space. In this way, the use of the contractions semigroup theory, see [38,39], to get the existence of the mixed problem solution and the uniqueness of this solution and, also, a result regarding the continuous dependence of the solution in relation to the initial data and loads, proves to be the most appropriate method.

\section{Notations and Basic Equations}

It is considered a porous micromorphic thermoelastic material, occupying the domain $\mathcal{D}$ in the three-dimensional Euclidean space $\mathbb{R}^{3}$, at the initial time $t_{0}, \overline{\mathcal{D}}$ and $\partial \mathcal{D}$ representing the notations corresponding to the closure of the regular region $\mathcal{D}$, respectively to the boundary, a smooth surface.

The material evolution is referred to a rectangular Cartesian fixed system of axes, each point of the domain being represented by three rectangular coordinates, noting that $x$ will be utilized for $\left(x_{1}, x_{2}, x_{3}\right)$ ant $t$ for the time. The functions that appear in the following, throughout this article, are considered to be functions of $(x, t)$, defined on the cylinder $\overline{\mathcal{D}} \times(0, \infty)$, where $\overline{\mathcal{D}}=\mathcal{D} \cup \partial \mathcal{D}$, with the specification that both arguments will be missing when there is no risk of confusion.

The vectors, tensors, as well as matrices will be represented by bold symbols, for example $\boldsymbol{u}=\left(u_{i}\right)_{1 \leq i \leq 3}, \boldsymbol{\varphi}=\left(\varphi_{i j}\right)_{1 \leq i, j \leq 3}$.

The equations describing the behavior of a porous micromorphic thermoelastic material with microtemperatures are expressed by means of the variables $\left(u_{i}, \varphi_{i j}, v, \alpha, \zeta_{i}\right), u_{i}$ being the displacement vector components, $\varphi_{i j}$ the microdeformation tensor components, $v$ the volume fraction directly related to the voids and $\alpha, \zeta_{i}$, the two new variables, introduced by the following relations

$$
\alpha(x, t)=\int_{t_{0}}^{t} \theta(x, s) d s, \quad \zeta_{i}(x, t)=\int_{t_{0}}^{t} \vartheta_{i}(x, s) d s .
$$

In the previous relation (1), $t_{0}$ represents the reference time, $\theta$ represents the temperature variation compared to $T_{0}$, the absolute temperature of the material in the reference configuration, 


$$
\theta(x, t)=T(x, t)-T_{0}
$$

and $\vartheta_{i}$ represent the variations of microtemperatures in relation to the reference configuration microtemperatures, denoted by $T_{i}^{o}$,

$$
\vartheta_{i}=T_{i}-T_{i}^{0} .
$$

The variable $\alpha$, defined with the help of the temperature, represented by the relation $(1)_{1}$, is called "the thermal displacement", its introduction being made by Green and Naghdi in [40] and the variables $\zeta_{i}$, represented by the relation $(1)_{2}$, are called "the microthermal displacements".

Regarding the coordinates of an arbitrary point of the body, they will be denoted by $\left(x_{i}\right)$, respectively, in the reference configuration, by $\left(x_{i}^{\prime}\right)$, therefore, it can be assumed that the material absolute temperature is represented as follows

$$
\theta+T_{i}\left(x_{i}-x_{i}^{\prime}\right)
$$

Throughout this article, the well-known Einstein summation convention will be applied in the case of index repetition and the Latin indices will take the values 1, 2, 3. A comma followed by an index represents the function partial differentiation with respect to the related Cartesian coordinate and a point above a function will represent the partial differentiation of that function corresponding to the time.

The fundamental equations and conditions that describe the behavior of an anisotropic, thermoelastic micromorphic material with voids and microtemperatures are presented below:

- the geometric equations, representing the strain tensors $\varepsilon_{i j}, \mu_{i j}, \gamma_{i j k}$, respectively the vector $v_{i}$, in compliance with [14],

$$
\varepsilon_{i j}=u_{j, i}-\varphi_{j i}, \quad \mu_{i j}=\frac{1}{2}\left(\varphi_{i j}+\varphi_{j i}\right), \gamma_{i j k}=\varphi_{i j, k}, v_{i}=v_{, i},(x, t) \in \mathcal{D} \times(0, \infty),
$$

- the equations of motion

$$
\begin{aligned}
& t_{j i, j}+\rho f_{i}=\rho \ddot{u}_{i}, \\
& m_{k i j, k}+t_{j i}-s_{j i}+\rho \ell_{i j}=I_{j k} \ddot{\varphi}_{i k},
\end{aligned}
$$

- the balance of equilibrated forces

$$
\lambda_{i, i}-\xi+\rho \ell=\rho k \ddot{\nu},
$$

- the energy equation, in the form of the following relation

$$
\rho \chi_{i}+r_{i}-h_{i}=0
$$

- the additional energy equations, as a result of the existence of the microtemperatures, introduced by the next relations, according to [40]

$$
\begin{aligned}
\rho \dot{\eta} & =r_{i, i}+\rho s, \\
\rho \dot{\eta}_{i} & =M_{j i, j}+\rho \mathcal{M}_{i},
\end{aligned}
$$


- the constitutive equations, obtained by applying the Ieșan and Quintanilla procedure, presented in [33], see also [27],

$$
\begin{aligned}
t_{i j} & =C_{i j m n} \varepsilon_{m n}+E_{i j m n} \mu_{m n}+F_{i j l m n} \gamma_{l m n}+B_{i j} v-a_{i j} \dot{\alpha}+d_{i j m n} \zeta_{m, n}, \\
s_{i j} & =E_{m n i j} \varepsilon_{m n}+B_{i j m n} \mu_{m n}+G_{i j l m n} \gamma_{l m n}+C_{i j} v-b_{i j} \dot{\alpha}+e_{i j m n} \zeta_{m, n}, \\
m_{k i j} & =A_{i j k l m n} \gamma_{l m n}+F_{l m i j k} \varepsilon_{l m}+G_{l m i j k} \mu_{l m}+D_{i j k} v-c_{i j k} \dot{\alpha}+f_{i j k m n} \zeta_{m, n}, \\
\rho \eta & =a_{i j} \varepsilon_{i j}+b_{i j} \mu_{i j}+c_{i j k} \gamma_{i j k}+v+a \dot{\alpha}+h_{i j} \zeta_{i, j}, \\
\xi & =B_{i j} \varepsilon_{i j}+C_{i j} \mu_{i j}+D_{i j k} \gamma_{i j k}+\sigma v-\dot{\alpha}+F_{i j} \zeta_{i, j}, \\
\lambda_{i} & =A_{i j} v_{j}-d_{i j} \dot{\zeta}_{j}+H_{i j} \alpha_{, j}, \\
\rho \eta_{i} & =d_{j i} v_{j}+D_{i j} \dot{\zeta}_{j}+E_{j i} \alpha_{, j}, \\
r_{i} & =H_{j i} v_{j}-E_{i j} \dot{\zeta}_{j}+K_{i j} \alpha_{, j}, \\
M_{i j} & =d_{i j m n} \varepsilon_{m n}+e_{i j m n} \mu_{m n}+f_{l m n i j} \gamma_{l m n}+g_{i j m n} \zeta_{m, n}+F_{i j} v-h_{i j} \dot{\alpha} .
\end{aligned}
$$

In the previous equations the following notations were used:

- $t_{i j}, s_{i j}, m_{k i j}$ are the stress tensor components, the microstress tensor components and the stress moment tensor components,

- $\quad \rho$ is the reference mass density,

- $f_{i}$ are the components of the body force vector,

- $\ell_{i j}$ are the body moment tensor components,

- $I_{j k}$ are the components of the microinertia tensor, endowed with the property of symmetry $I_{j k}=I_{k j}$,

- $\lambda_{i}$ are the equilibrated stress vector components related to the voids,

- $\xi$ represents the intrinsic equilibrated body force,

- $\quad \ell$ represents the extrinsic equilibrated body force corresponding to the voids,

- $k$ is the equilibrated inertia coefficient,

- $\chi_{i}$ represents the internal rate of entropy production per mass unit,

- $r_{i}$ are the entropy flux vector components,

- $h_{i}$ are the mean entropy flux vector components,

- $\quad \eta$ represents the specific entropy per mass unit,

- $s$ represents the external rate of entropy supply,

- $\eta_{i}$ are the first entropy moment vector components,

- $M_{i j}$ are the first entropy flux moment tensor components,

- $\mathcal{M}_{i}$ are first moment of the external rate of entropy supply.

The constitutive coefficients, which appear in the representation of the constitutive Equation (7), express the material specifities, and are prescribed functions, of class $C^{1}(\mathcal{D})$, which fulfill the following symmetry relations

$$
\begin{aligned}
& C_{i j k l}=C_{k l i j}, B_{i j k l}=B_{k l i j}=B_{j i k l}, A_{i j k l m n}=A_{l m n i j k}, E_{i j k l}=E_{k l i j}, \\
& G_{i j l m n}=G_{j i l m n}, A_{i j}=A_{j i}, B_{i j}=B_{j i}, C_{i j}=C_{j i}, K_{i j}=K_{j i}, D_{i j}=D_{j i}, \\
& a_{i j}=a_{j i}, c_{i j k}=c_{j i k}, e_{i j m n}=e_{j i m n}, g_{i j m n}=g_{m n i j}, d_{i j m n}=d_{j i m n} .
\end{aligned}
$$


The use of the geometric Equation (2) and the constitutive Equation (7) into the equations of motion (3), the balance of equilibrated forces (4), as well as into the additional equations of energy (6), leads to a system of differential equations, with the unknowns $u_{i}, \varphi_{i j}, \nu, \alpha$ and $\zeta_{i}$, having the form

$$
\begin{aligned}
& {\left[C_{i j m n}\left(u_{n, m}-\varphi_{n m}\right)+E_{i j m n} \varphi_{n m}+F_{i j l m n} \varphi_{l m, n}+B_{i j} v-a_{i j} \dot{\alpha}+d_{i j m n} \zeta_{m, n}\right]_{, j}+\rho f_{i}=\rho \ddot{u}_{i},} \\
& {\left[A_{i j k l m n} \varphi_{l m, n}+F_{l m i j k}\left(u_{m, l}-\varphi_{m l}\right)+G_{l m i j k} \varphi_{m l}+D_{i j k} v-c_{i j k} \dot{\alpha}+f_{i j k m n} \zeta_{m, n}\right]_{, k}+} \\
& +\left[C_{i j m n}\left(u_{n, m}-\varphi_{n m}\right)+E_{i j m n} \varphi_{n m}+F_{i j l m n} \varphi_{l m, n}+B_{i j} v-a_{i j} \dot{\alpha}+d_{i j m n} \zeta_{m, n}\right]- \\
& -\left[E_{m n i j}\left(u_{n, m}-\varphi_{n m}\right)+B_{i j m n} \varphi_{n m}+G_{i j l m n} \varphi_{l m, n}+C_{i j} v-b_{i j} \dot{\alpha}+e_{i j m n} \zeta_{m, n}\right]+\rho \ell_{i j}=I_{j k} \ddot{\varphi}_{i k}, \\
& \left(A_{i j} v_{j}-d_{i j} \dot{\zeta}_{j}+H_{i j} \alpha_{, j}\right)_{, i}-B_{i j}\left(u_{j, i}-\varphi_{j i}\right)-C_{i j} \varphi_{j i}-D_{i j k} \varphi_{i j, k}-\sigma v+\dot{\alpha}-F_{i j} \zeta_{i, j}+\rho \ell=\rho k \ddot{v}, \\
& \left(H_{j i} v_{j}-E_{i j} \dot{\zeta}_{j}+K_{i j} \alpha_{, j}\right)_{, i}-a_{i j}\left(\dot{u}_{j, i}-\dot{\varphi}_{j i}\right)-b_{i j} \dot{\varphi}_{j i}-c_{i j k} \dot{\varphi}_{i j, k}-\dot{v}-a \ddot{\alpha}-h_{i j} \dot{\zeta}_{i, j}=-\rho s, \\
& {\left[d_{i j m n}\left(u_{n, m}-\varphi_{n m}\right)+e_{i j m n} \varphi_{n m}+f_{l m n i j} \varphi_{l m, n}+g_{i j m n} \zeta_{m, n}+F_{i j} v-h_{i j} \dot{\alpha}\right]_{, j}-} \\
& -\left(d_{i j} \dot{v}_{j}+D_{i j} \ddot{\zeta}_{j}+E_{j i} \dot{\alpha}_{, j}\right)=-\rho \mathcal{M}_{i} .
\end{aligned}
$$

In accordance with the Dirichlet problem, corresponding to the system (9), the boundary conditions are considered as follows

$$
\begin{aligned}
u_{i}(x, t) & =\widetilde{u}_{i}(x, t), \\
\varphi_{i j}(x, t) & =\widetilde{\varphi}_{i j}(x, t), \\
v(x, t) & =\widetilde{v}(x, t), \quad(x, t) \in \partial \mathcal{D} \times(0, \infty), \\
\alpha(x, t) & =\widetilde{\alpha}(x, t), \\
\zeta_{i}(x, t) & =\widetilde{\zeta}_{i}(x, t),
\end{aligned}
$$

where $\widetilde{u}_{i}, \widetilde{\varphi}_{i j}, \widetilde{v}, \widetilde{\alpha}$ and $\widetilde{\zeta}_{i}$ are prescribed functions.

The initial conditions, which will be included in the mixed problem, corresponding to the above system of Equation (9), are presented below

$$
\begin{aligned}
u_{i}(x, 0) & =u_{i}^{0}(x), & \dot{u}_{i}(x, 0) & =u_{i}^{1}(x), \\
\varphi_{i j}(x, 0) & =\varphi_{i j}^{0}(x), & \dot{\varphi}_{i j}(x, 0) & =\varphi_{i j}^{1}(x), \\
v(x, 0) & =v^{0}(x), & \dot{v}(x, 0) & =v^{1}(x), \\
\alpha(x, 0) & =\alpha^{0}(x), & \dot{\alpha}(x, 0) & =\alpha^{1}(x), \\
\zeta_{i}(x, 0) & =\zeta_{i}^{0}(x), & \dot{\zeta}_{i}(x, 0) & =\zeta_{i}^{1}(x),
\end{aligned}
$$

for any $x \in \mathcal{D}, u_{i}^{0}, u_{i}^{1}, \varphi_{i j}^{0}, \varphi_{i j}^{1}, v^{0}, v^{1}, \alpha^{0}, \alpha^{1}, \zeta_{i}^{0}$ and $\zeta_{i}^{1}$ also being prescribed functions.

The functions and conditions presented above are assumed to be sufficiently regular on their definition domain to be able to realize subsequent mathematical operations.

In the theory of the thermoelastic porous micromorphic materials having microtemperatures, the ordered system $\left(u_{i}, \varphi_{i j}, v, \alpha, \zeta_{i}\right)$ is a solution of the mixed initial-boundary value problem, denoted by $\mathcal{P}$, if it satisfies the system of Equation (9), together with the initial conditions (11) and the boundary conditions (10), for all $(x, t) \in \mathcal{D} \times(0, \infty)$.

\section{Results}

\subsection{Auxiliary Results}

The following theorem expresses an auxiliary result, useful for obtaining the uniqueness of the mixed problem solution of the thermoelastic porous micromorphic materials. 
Theorem 1. The variables that describe the deformation of a thermoelastic porous micromorphic material with microtemperatures verify the following equality

$$
\begin{aligned}
& t_{i j} \varepsilon_{i j}+s_{i j} \mu_{i j}+m_{k i j} \gamma_{i j k}+\rho \eta \dot{\alpha}+\xi v+\lambda_{i} v_{i}+\rho \eta_{i} \dot{\zeta}_{i}+r_{i} \alpha_{, i}+M_{i j} \zeta_{i, j}= \\
& =C_{i j m n} \varepsilon_{i j} \varepsilon_{m n}+2 E_{i j m n} \varepsilon_{i j} \mu_{m n}+2 F_{i j l m n} \varepsilon_{i j} \gamma_{l m n}+B_{i j m n} \mu_{i j} \mu_{m n}+ \\
& +2 G_{i j l m n} \mu_{i j} \gamma_{l m n}+A_{i j k l m n} \gamma_{i j k} \gamma_{l m n}+2 B_{i j} \varepsilon_{i j} v+2 C_{i j} \mu_{i j} v+2 D_{i j k} \gamma_{i j k} v+ \\
& +2 F_{i j} \zeta_{i, j} v+\sigma v^{2}+2 d_{i j m n} \varepsilon_{i j} \zeta_{m, n}+2 e_{i j m n} \mu_{i j} \zeta_{m, n}+2 f_{i j k m n} \gamma_{i j k} \zeta_{m, n}+ \\
& +A_{i j} v_{i} v_{j}+2 H_{i j} v_{i} \alpha_{, j}+K_{i j} \alpha_{, i} \alpha_{, j}+g_{i j m n} \zeta_{i, j} \zeta_{m, n}+D_{i j} \dot{\zeta}_{i} \dot{\zeta}_{j}+a(\dot{\alpha})^{2} .
\end{aligned}
$$

Proof. Multiplying the first constitutive Equation $(7)_{1}$ by $\varepsilon_{i j}$, the second $(7)_{2}$ by $\mu_{i j}$, the third $(7)_{3}$ by $\gamma_{i j k}$, the Equation $(7)_{4}$ by $\dot{\alpha},(7)_{5}$ by $v$, the sixth constitutive Equation $(7)_{6}$ by $v_{, i},(7)_{7}$ by $\dot{\zeta}_{i}$ and the last two constitutive Equations $(7)_{8}$ and $(7)_{9}$ by $\alpha_{, i}$, respectively by $\zeta_{i, j}$, then making the sum of the new obtained relations and applying the symmetry relations (8), the relation (12), representing the conclusion of the Theorem 1, is deduced. We must specify that the above multiplications are chosen for having the unit of energy density. Thus, the proof of Theorem 1 is complete.

The free energy $\Psi$ is considered in the quadratic form below

$$
\begin{aligned}
\Psi & =\frac{1}{2} C_{i j m n} \varepsilon_{i j} \varepsilon_{m n}+E_{i j m n} \varepsilon_{i j} \mu_{m n}+F_{i j l m n} \varepsilon_{i j} \gamma_{l m n}+\frac{1}{2} B_{i j m n} \mu_{i j} \mu_{m n}+ \\
& +G_{i j l m n} \mu_{i j} \gamma_{l m n}+\frac{1}{2} A_{i j k l m n} \gamma_{i j k} \gamma_{l m n}+B_{i j} \varepsilon_{i j} v+C_{i j} \mu_{i j} v+D_{i j k} \gamma_{i j k} v+ \\
& +F_{i j} \zeta_{i, j} v+\frac{1}{2} \sigma v^{2}+d_{i j m n} \varepsilon_{i j} \zeta_{m, n}+e_{i j m n} \mu_{i j} \zeta_{m, n}+f_{i j k m n} \gamma_{i j k} \zeta_{m, n}+ \\
& +\frac{1}{2} A_{i j} v_{i} v_{j}+H_{i j} v_{i} \alpha_{, j}+\frac{1}{2} K_{i j} \alpha_{, i} \alpha_{, j}+\frac{1}{2} g_{i j m n} \zeta_{i, j} \zeta_{m, n} .
\end{aligned}
$$

In order to obtain the result regarding the uniqueness of the solution of the mixed problem $\mathcal{P}$, the following standard hypotheses are assumed:

(i) $\rho>0, I_{i j}>0, k>0, a>0$;

(ii) the quadratic form $\Psi$, expressed through the relation (13), is positively semi-defined;

(iii) the conductivity coefficients $K_{i j}$ and the constitutive ones $D_{i j}$ are the components of positively defined tensors, i.e., $\exists k_{0}>0$ and $d_{0}>0$ such that

$$
K_{i j} \xi_{i} \xi_{j} \geq k_{0} \xi_{i} \xi_{i}, D_{i j} \xi_{i} \xi_{j} \geq d_{0} \xi_{i} \xi_{i}, \forall \xi_{i}
$$

\subsection{Main Results}

\subsubsection{Uniqueness}

Theorem 2. If both the symmetry relations (8) and the standard hypotheses (i)-(iii) are verified, then the mixed initial-boundary value problem $\mathcal{P}$ has at most one solution.

Proof. Making an analogy with the method used to prove the Theorem 1, we multiply the first constitutive Equation $(7)_{1}$ by $\dot{\varepsilon}_{i j},(7)_{2}$ by $\dot{\mu}_{i j},(7)_{3}$ by $\dot{\gamma}_{i j k}$, the constitutive Equation $(7)_{5}$ by $\dot{v},(7)_{6}$ by $\dot{v}_{, i}$, the Equations $(7)_{8}$ and $(7)_{9}$ by $\dot{\alpha}_{, i}$, respectively by $\dot{\zeta}_{i, j}$, and the derivatives, in relation to time, 
of the constitutive Equations $(7)_{4}$ and $(7)_{7}$ by $\dot{\alpha}$, respectively $\dot{\zeta}_{i}$, then we add, member by member, the relations thus obtained and through the symmetry relations (8) we deduce the next relation:

$$
\begin{aligned}
& t_{i j} \dot{\varepsilon}_{i j}+s_{i j} \dot{\mu}_{i j}+m_{k i j} \dot{\gamma}_{i j k}+\rho \dot{\gamma} \dot{\alpha}+\xi \dot{v}+\lambda_{i} \dot{v}_{, i}+\rho \dot{\eta}_{i} \dot{\zeta}_{i}+r_{i} \dot{\alpha}_{, i}+M_{i j} \dot{\zeta}_{i, j}= \\
& =C_{i j m n} \dot{\varepsilon}_{i j} \varepsilon_{m n}+E_{i j m n}\left(\dot{\varepsilon}_{i j} \mu_{m n}+\varepsilon_{i j} \dot{\mu}_{m n}\right)+F_{i j l m n}\left(\dot{\varepsilon}_{i j} \gamma_{l m n}+\varepsilon_{i j} \dot{\gamma}_{l m n}\right)+ \\
& +B_{i j m n} \dot{\mu}_{i j} \mu_{m n}+G_{i j l m n}\left(\dot{\mu}_{i j} \gamma_{l m n}+\mu_{i j} \dot{\gamma}_{l m n}\right)+A_{i j k l m n} \dot{\gamma}_{i j k} \gamma_{l m n}+B_{i j}\left(\dot{\varepsilon}_{i j} v+\varepsilon_{i j} \dot{v}\right)+ \\
& +C_{i j}\left(\dot{\mu}_{i j} v+\mu_{i j} \dot{v}\right)+D_{i j k}\left(\dot{\gamma}_{i j k} v+\gamma_{i j k} \dot{v}\right)+F_{i j}\left(\dot{\zeta}_{i, j} v+\zeta_{i, j} \dot{v}\right)+\sigma v \dot{v}+ \\
& +d_{i j m n}\left(\dot{\varepsilon}_{i j} \zeta_{m, n}+\varepsilon_{i j} \dot{\zeta}_{m, m}\right)+e_{i j m n}\left(\dot{\mu}_{i j} \zeta_{m, n}+\mu_{i j} \dot{\zeta}_{m, n}\right)+f_{i j k m n}\left(\dot{\gamma}_{i j k} \zeta_{m, n}+\gamma_{i j k} \dot{\zeta}_{m, n}\right)+ \\
& +A_{i j} \dot{v}_{i} v_{j}+H_{i j}\left(\dot{v}_{i} \alpha_{, j}+v_{i} \dot{\alpha}_{, j}\right)+K_{i j} \dot{\alpha}_{, i} \alpha_{, j}+g_{i j m n} \dot{\zeta}_{i, j} \zeta_{m, n}+D_{i j} \dot{\zeta}_{i} \ddot{\zeta}_{j}+a \dot{\alpha} \ddot{\alpha} .
\end{aligned}
$$

The previous relation (14) can be reformulated as follows

$$
\begin{aligned}
& t_{i j} \dot{\varepsilon}_{i j}+s_{i j} \dot{\mu}_{i j}+m_{k i j} \dot{\gamma}_{i j k}+\rho \dot{\eta} \dot{\alpha}+\xi \dot{v}+\lambda_{i} \dot{v}_{i}+\rho \dot{\eta}_{i} \dot{\zeta}_{i}+r_{i} \dot{\alpha}_{, i}+M_{i j} \dot{\zeta}_{i, j}= \\
& =\frac{\partial}{\partial t}\left(\frac{1}{2} C_{i j m n} \varepsilon_{i j} \varepsilon_{m n}+E_{i j m n} \varepsilon_{i j} \mu_{m n}+F_{i j l m n} \varepsilon_{i j} \gamma_{l m n}+\frac{1}{2} B_{i j m n} \mu_{i j} \mu_{m n}+\right. \\
& +G_{i j l m n} \mu_{i j} \gamma_{l m n}+\frac{1}{2} A_{i j k l m n} \gamma_{i j k} \gamma_{l m n}+B_{i j} \varepsilon_{i j} v+C_{i j} \mu_{i j} v+D_{i j k} \gamma_{i j k} v+ \\
& +F_{i j} \zeta_{i, j} v+\frac{1}{2} \sigma v^{2}+d_{i j m n} \varepsilon_{i j} \zeta_{m, n}+e_{i j m n} \mu_{i j} \zeta_{m, n}+f_{i j k m n} \gamma_{i j k} \zeta_{m, n}+ \\
& \left.+\frac{1}{2} A_{i j} v_{i} v_{j}+H_{i j} v_{i} \alpha_{, j}+\frac{1}{2} K_{i j} \alpha_{, i} \alpha_{, j}+\frac{1}{2} g_{i j m n} \zeta_{i, j} \zeta_{m, n}+\frac{1}{2} \dot{\zeta}_{i} \dot{\zeta}_{j}+\frac{1}{2} a(\dot{\alpha})^{2}\right)= \\
& =\frac{\partial \Psi}{\partial t}+\frac{1}{2} \frac{\partial}{\partial t}\left[D_{i j} \dot{\zeta}_{i} \dot{\zeta}_{j}+a(\dot{\alpha})^{2}\right] .
\end{aligned}
$$

At the same time, taking into account the geometric Equation (2), the equations of motion (3), the equilibrated forces balance (4), as well as the additional equations of energy (6), it follows that

$$
\begin{aligned}
& t_{i j} \dot{\varepsilon}_{i j}+s_{i j} \dot{\mu}_{i j}+m_{k i j} \dot{\gamma}_{i j k}+\rho \dot{\eta} \dot{\alpha}+\xi \dot{v}+\lambda_{i} \dot{v}_{i}+\rho \dot{\eta}_{i} \dot{\zeta}_{i}+r_{i} \dot{\alpha}_{, i}+M_{i j} \dot{\zeta}_{i, j}= \\
& =\left(t_{i j} \dot{u}_{j}+m_{i j k} \dot{\varphi}_{j k}+\lambda_{i} \dot{v}+r_{i} \dot{\alpha}+M_{i j} \dot{\zeta}_{j}\right)_{, i}+\rho\left(f_{i} \dot{u}_{i}+\ell_{i j} \dot{\varphi}_{i j}+\ell \dot{v}+s \dot{\alpha}+\mathcal{M}_{i} \dot{\zeta}_{i}\right)- \\
& -\rho \dot{u}_{i} \ddot{u}_{i}-I_{j k} \dot{\varphi}_{i j} \ddot{\varphi}_{i k}-\rho k \dot{v} \ddot{v} .
\end{aligned}
$$

Using the relations (15) and (16), the equality below is obtained

$$
\begin{aligned}
& \left(t_{i j} \dot{u}_{j}+m_{i j k} \dot{\varphi}_{j k}+\lambda_{i} \dot{v}+r_{i} \dot{\alpha}+M_{i j} \dot{\zeta}_{j}\right)_{, i}+\rho\left(f_{i} \dot{u}_{i}+\ell_{i j} \dot{\varphi}_{i j}+\ell \dot{v}+s \dot{\alpha}+\mathcal{M}_{i} \dot{\zeta}_{i}\right)= \\
& =\frac{\partial \Psi}{\partial t}+\frac{1}{2} \frac{\partial}{\partial t}\left[\rho \dot{u}_{i} \dot{u}_{i}+I_{j k} \dot{\varphi}_{i j} \dot{\varphi}_{i k}+\rho k(\dot{v})^{2}+D_{i j} \dot{\zeta}_{i} \dot{\zeta}_{j}+a(\dot{\alpha})^{2}\right] .
\end{aligned}
$$

By integrating the previous relation (17) on $\mathcal{D}$ and by applying the divergence theorem, a fundamental result is obtained for proving the mixed initial-boundary value problem $\mathcal{P}$, the result consisting in the following equality:

$$
\begin{aligned}
& \int_{\mathcal{D}} \rho\left(f_{i} \dot{u}_{i}+\ell_{i j} \dot{\varphi}_{i j}+\ell \dot{v}+s \dot{\alpha}+\mathcal{M}_{i} \dot{\zeta}_{i}\right) d V+ \\
& +\int_{\partial \mathcal{D}}\left(t_{i j} \dot{u}_{j}+m_{i j k} \dot{\varphi}_{j k}+\lambda_{i} \dot{v}+r_{i} \dot{\alpha}+M_{i j} \dot{\zeta}_{j}\right) n_{i} d A= \\
& =\frac{\partial}{\partial t} \int_{\mathcal{D}}\left\{\Psi+\frac{1}{2}\left[\rho \dot{u}_{i} \dot{u}_{i}+I_{j k} \dot{\varphi}_{i j} \dot{\varphi}_{i k}+\rho k(\dot{v})^{2}+D_{i j} \dot{\zeta}_{i} \dot{\zeta}_{j}+a(\dot{\alpha})^{2}\right]\right\} d V .
\end{aligned}
$$

The demonstration of the uniqueness of the mixed problem solution is made assuming that the mixed problem $\mathcal{P}$ admits two solutions $s^{\prime}=\left(u_{i}^{\prime}, \varphi_{i j}^{\prime}, v^{\prime}, \alpha^{\prime}, \zeta_{i}^{\prime}\right)$ and $s^{\prime \prime}=\left(u_{i}^{\prime \prime}, \varphi_{i j}^{\prime \prime}, v^{\prime \prime}, \alpha^{\prime \prime}, \zeta_{i}^{\prime \prime}\right)$, 
the difference of the solutions, $s^{D}=s^{\prime \prime}-s^{\prime}$ being, in turn, a solution of the mixed problem $\mathcal{P}$, based on linearity.

Using the notations below

$$
\begin{aligned}
& u_{i}^{D}=u_{i}^{\prime \prime}-u_{i,}^{\prime}, \\
& \varphi_{i j}^{D}=\varphi_{i j}^{\prime \prime}-\varphi_{i j}^{\prime}, \\
& v^{D}=v^{\prime \prime}-v^{\prime}, \\
& \alpha^{D}=\alpha^{\prime \prime}-\alpha^{\prime}, \\
& \zeta_{i}^{D}=\zeta_{i}^{\prime \prime}-\zeta_{i}^{\prime},
\end{aligned}
$$

and the quantities corresponding to these differences, which will also be denoted by ${ }^{D}$, it can be noted that the solution

$$
s^{D}=\left(u_{i}^{D}, \varphi_{i j}^{D}, v^{D}, \alpha^{D}, \zeta_{i}^{D}\right)
$$

satisfies the equations of motion (3), the balance of equilibrated forces (4) and the equations of energy (6), in the circumstances of null loads and conditions, both initial and boundary conditions become homogeneous, as follows

$$
\begin{array}{ll}
u_{i}^{D}(x, 0)=0, & \dot{u}_{i}^{D}(x, 0)=0, \\
\varphi_{i j}^{D}(x, 0)=0, & \dot{\varphi}_{i j}^{D}(x, 0)=0, \\
v^{D}(x, 0)=0, & \dot{v}^{D}(x, 0)=0, \quad x \in \mathcal{D}, \\
\alpha^{D}(x, 0)=0, & \dot{\alpha}^{D}(x, 0)=0, \\
\zeta_{i}^{D}(x, 0)=0, & \dot{\zeta}_{i}^{D}(x, 0)=0,
\end{array}
$$

and

$$
\widetilde{u}_{i}=0, \widetilde{\varphi}_{i j}=0, \widetilde{v}=0, \widetilde{\alpha}=0, \widetilde{\zeta}_{i}=0, \text { pe } \partial \mathcal{D} \times(0, \infty),
$$

mentioning that

$$
\begin{array}{rlrl}
\varepsilon_{i j}^{D}(x, 0) & =0, & \mu_{i j}^{D}(x, 0)=0, & \gamma_{i j k}^{D}(x, 0)=0, \\
v_{, i}^{D}(x, 0)=0, & \alpha_{, i}^{D}(x, 0)=0, & \zeta_{i, j}^{D}(x, 0)=0, x \in \mathcal{D} .
\end{array}
$$

Taking these considerations into account, the relation (18) leads to the next equality

$$
\int_{\mathcal{D}}\left[\rho \dot{u}_{i}^{D} \dot{u}_{i}^{D}+I_{j k} \dot{\varphi}_{i j}^{D} \dot{\varphi}_{i k}^{D}+\rho k\left(\dot{v}^{D}\right)^{2}+D_{i j} \dot{\zeta}_{i}^{D} \dot{\zeta}_{j}^{D}+a\left(\dot{\alpha}^{D}\right)^{2}+2 \Psi^{D}\right] d V=0, t \geq 0 .
$$

In the conditions of the standard hypothesis (ii), of the specification (23), as well as of the observation that the quadratic form $\Psi$, represented by the relation (13), corresponding to the differences is null, the previous relation (24) becomes

$$
\int_{\mathcal{D}}\left[\rho \dot{u}_{i}^{D} \dot{u}_{i}^{D}+I_{j k} \dot{\varphi}_{i j}^{D} \dot{\varphi}_{i k}^{D}+\rho k\left(\dot{v}^{D}\right)^{2}+D_{i j} \dot{\zeta}_{i}^{D} \dot{\zeta}_{j}^{D}+a\left(\dot{\alpha}^{D}\right)^{2}\right] d V=0 .
$$

By considering the standard assumptions ( $i$ ) and (iii) regarding the coefficients $\rho, I_{i j}, k, a$ and the tensor $D_{i j}$, the relation (25) leads to the conclusion

$$
\dot{u}_{i}^{D}=0, \dot{\varphi}_{i j}^{D}=0, \dot{v}^{D}=0, \dot{\alpha}^{D}=0, \dot{\zeta}_{i}^{D}=0, \text { pe } \mathcal{D} \times(0, \infty),
$$

and the relation (21) ensures that 


$$
u_{i}^{D}(x, t)=0, \varphi_{i j}^{D}(x, t)=0, v^{D}(x, t)=0, \alpha^{D}(x, t)=0, \zeta_{i}^{D}(x, t)=0,(x, t) \in \mathcal{D} \times(0, \infty),
$$

thus concluding that the solution to the mixed problem is unique. In this way, the proof of the Theorem 2 is concluded.

\subsubsection{Existence}

The study on the existence of the solution of the mixed initial-boundary value problem will be approached in the context of homogeneous boundary conditions, which means that

$$
\widetilde{u}_{i}(x, t)=0, \widetilde{\varphi}_{i j}(x, t)=0, \widetilde{v}(x, t)=0, \widetilde{\alpha}(x, t)=0, \widetilde{\zeta}_{i}(x, t)=0, \quad(x, t) \in \mathcal{D} \times(0, \infty) .
$$

The fact that both the system of equations and the conditions associated with the mixed problem are extremely complex, the demonstration of the theorem with respect to the existence of the solution to this problem will be made in a special way, turning the mixed problem $\mathcal{P}$ into a problem of Cauchy type, correlated with an evolutionary equation defined on Hilbert space that has been properly chosen, see $[27,31,32]$.

In the following, the well-known Sobolev and Hilbert spaces will be used, more details on these spaces can be find in [41-43].

Based on classic Hilbert spaces $W_{0}^{1,2}$ and $L^{2}$, the Hilbert space $\mathcal{H}$ is defined as follows

$$
\mathcal{H}=\boldsymbol{W}_{0}^{1,2} \times L^{2} \times \boldsymbol{W}_{0}^{1,2} \times L^{2} \times W_{0}^{1,2} \times L^{2} \times W_{0}^{1,2} \times L^{2} \times W_{0}^{1,2} \times L^{2},
$$

where

$$
W_{0}^{1,2}=W_{0}^{1,2} \times W_{0}^{1,2} \times W_{0}^{1,2}=\left[W_{0}^{1,2}\right]^{3}, \quad L^{2}=L^{2} \times L^{2} \times L^{2}=\left[L^{2}\right]^{3} .
$$

In the previous relation, the Hilbert spaces that are found in the composition of the definition product induce a norm that endows the Hilbert space $\mathcal{H}$, further referring to it as the original norm of the space $\mathcal{H}$.

The following scalar product is defined on the space $\mathcal{H}$ :

$$
\begin{aligned}
& <\left(u_{i}, U_{i}, \varphi_{i j}, \Phi_{i j}, v, \mu, \alpha, \beta, \zeta_{i}, \zeta_{i}\right),\left(u_{i}^{\prime}, U_{i}^{\prime}, \varphi_{i j}^{\prime}, \Phi_{i j}^{\prime}, v^{\prime}, \mu^{\prime}, \alpha^{\prime}, \beta^{\prime}, \zeta_{i}^{\prime}, \zeta_{i}^{\prime}\right)>= \\
& \frac{1}{2} \int_{\mathcal{D}}\left(\rho U_{i} U_{i}^{\prime}+I_{j k} \Phi_{i j} \Phi_{i k}^{\prime}+\rho k \mu \mu^{\prime}+D_{i j} \zeta_{i} \zeta_{j}^{\prime}+a \beta \beta^{\prime}\right) d V+\frac{1}{2} \int_{\mathcal{D}}\left[C_{i j m n} \varepsilon_{i j} \varepsilon_{m n}^{\prime}+\right. \\
& +E_{i j m n}\left(\varepsilon_{i j} \mu_{m n}^{\prime}+\varepsilon_{i j}^{\prime} \mu_{m n}\right)+F_{i j l m n}\left(\varepsilon_{i j} \gamma_{l m n}^{\prime}+\varepsilon_{i j}^{\prime} \gamma_{l m n}\right)+B_{i j m n} \mu_{i j} \mu_{m n}^{\prime}+ \\
& +G_{i j l m n}\left(\mu_{i j} \gamma_{l m n}^{\prime}+\mu_{i j}^{\prime} \gamma_{l m n}\right)+A_{i j k l m n} \gamma_{i j k} \gamma_{l m n}^{\prime}+B_{i j}\left(\varepsilon_{i j} v^{\prime}+\varepsilon_{i j}^{\prime} v\right)+C_{i j}\left(\mu_{i j} v^{\prime}+\mu_{i j}^{\prime} v\right)+ \\
& +D_{i j k}\left(\gamma_{i j k} v^{\prime}+\gamma_{i j k}^{\prime} v\right)+F_{i j}\left(\zeta_{i, j} v^{\prime}+\zeta_{i, j}^{\prime} v\right)+\sigma v v^{\prime}+d_{i j m n}\left(\varepsilon_{i j} \zeta_{m, n}^{\prime}+\varepsilon_{i j}^{\prime} \zeta_{m, n}\right)+ \\
& +e_{i j m n}\left(\mu_{i j} \zeta_{m, n}^{\prime}+\mu_{i j}^{\prime} \zeta_{m, n}\right)+f_{i j k m n}\left(\gamma_{i j k} \zeta_{m, n}^{\prime}+\gamma_{i j k}^{\prime} \zeta_{m, n}\right)+A_{i j} v_{i} v_{j}^{\prime}+ \\
& \left.+H_{i j}\left(v_{i} \alpha_{, j}^{\prime}+v_{i}^{\prime} \alpha_{, j}\right)+K_{i j} \alpha_{, i} \alpha_{, j}^{\prime}+g_{i j m n} \zeta_{i, j} \zeta_{m, n}^{\prime}\right] .
\end{aligned}
$$

Theorem 3. The scalar product introduced by the relation (28) induces a norm equivalent to the original norm in the Hilbert space $\mathcal{H}$.

Proof. Considering both Korn's first inequality, see [44-46], and the standard hypotheses, the conclusion of the Theorem 3 can be deduced. 
The choice of the following operators form is inspired by the functions that form part of the left member of each equation that constitutes the system (9), so that, applying the procedure presented in [27], the operators below are introduced:

$$
\begin{aligned}
C_{i}^{1} \boldsymbol{u} & =\frac{1}{\rho} C_{i j m n} u_{n, m j}, \quad C_{i}^{2} \boldsymbol{\varphi}=-\frac{1}{\rho}\left[\left(C_{i j m n}-E_{i j m n}\right) \varphi_{n m, j}-F_{i j l m n} \varphi_{l m, n j}\right], \\
B_{i} v & =\frac{1}{\rho} B_{i j} v_{, j}, \quad A_{i}^{1} \beta=-\frac{1}{\rho} a_{i j} \beta_{, j} \quad D_{i}^{1} \zeta=\frac{1}{\rho} d_{i j m n} \zeta_{m, n j}, \\
C_{k i}^{3} \boldsymbol{u} & =\left(I_{j k}\right)^{-1}\left[\left(C_{i j m n}-E_{m n i j}\right) u_{n, m}+F_{l m i j k} u_{m, l k}\right], \\
C_{k i}^{4} \boldsymbol{\varphi} & =-\left(I_{j k}\right)^{-1}\left[\left(C_{i j m n}+B_{i j m n}-2 E_{i j m n}\right) \varphi_{n m}-A_{i j k l m n} \varphi_{l m, n k}\right], \\
B_{k i}^{2} v & =\left(I_{j k}\right)^{-1}\left[\left(B_{i j}-C_{i j}\right) v+D_{i j k} v_{, k}\right], \quad A_{k i}^{2} \beta=-\left(I_{j k}\right)^{-1}\left[\left(a_{i j}-b_{i j}\right) \beta+c_{i j k} \beta_{, k}\right], \\
D_{k i}^{2} \zeta & =-\left(I_{j k}\right)^{-1}\left[\left(e_{i j m n}-d_{i j m n}\right) \zeta_{m, n}-f_{i j k m n} \zeta_{m, n k}\right], \\
E v & =\frac{1}{\rho k}\left(A_{i j} v_{, i j}-\sigma v\right), \quad F \boldsymbol{s}=-\frac{1}{\rho k} d_{i j} \zeta_{j, i}, \quad G \alpha=\frac{1}{\rho k} H_{i j} \alpha_{, i j}, \\
H \boldsymbol{u} & =-\frac{1}{\rho k} B_{i j} u_{j, i}, \quad K \boldsymbol{\varphi}=\frac{1}{\rho k}\left[\left(B_{i j}+C_{i j}\right) \varphi_{j i}-D_{i j k} \varphi_{i j, k}\right], \\
L \beta & =\frac{1}{\rho k} \beta, \quad M \zeta=-\frac{1}{\rho k} F_{i j} \zeta_{i, j}, \quad N \alpha=\frac{1}{a} K_{i j} \alpha_{, i j,}, \\
P v & =\frac{1}{a} H_{j i} v_{i j}, \quad Q \zeta=-\frac{1}{a} e_{i j} \zeta_{j, i}, \quad R^{1} \boldsymbol{U}=-\frac{1}{a} a_{i j} U_{j, i}, \\
R^{2} \boldsymbol{\Phi} & =\frac{1}{a}\left[\left(a_{i j}-b_{i j}\right) \Phi_{j i}-c_{i j k} \Phi_{i j, k}\right], \quad S \mu=-\frac{1}{a} \mu, \quad C_{s}^{5} \boldsymbol{u}=\Lambda_{s i} d_{i j m n} u_{n, m j}, \\
C_{s}^{6} \boldsymbol{\varphi} & =\Lambda_{s i}\left[\left(e_{i j m n}-d_{i j m n}\right) \varphi_{n, m j}+f_{l m n i j} \varphi_{l m, n j}\right], \quad W_{s} v=\Lambda_{s i} F_{i j} v_{, j}, \\
T_{s} \beta & =-\Lambda_{s i} e_{i j} \beta_{, j}, \quad V_{s} \boldsymbol{\zeta}=\Lambda_{s i} g_{i j m n} \zeta_{m, n j}, \quad Z_{s} \mu=-\Lambda_{s i} d_{i j} \mu_{, j},
\end{aligned}
$$

where $e_{i j}=E_{i j}+h_{i j}$, and the matrix $\Lambda_{s i}=\left(\Lambda_{i j}\right)$ is introduced so that

$$
\Lambda_{i j} D_{i l}=\delta_{j l}
$$

$\delta_{j l}$ identity being the symbol of Kronecker.

In the following, the matrix functions $\mathcal{U}$ and $\mathcal{U}_{0}$, corresponding to the unknowns $u_{i}, \mathcal{U}_{i}$, $\varphi_{i j}, \ldots, \zeta_{i}, \varsigma_{i}$, and the initial data $u_{i}^{0}, U_{i}^{0}, \varphi_{i j}^{0}, \ldots, \zeta_{i}^{0}, \varsigma_{i}^{0}$, are considered, as well as the matrix corresponding to the loads, $\mathcal{F}$, in the form

$$
\begin{aligned}
\mathcal{U} & =\left(u_{i}, U_{i}, \varphi_{i j}, \Phi_{i j}, v, \mu, \alpha, \beta, \zeta_{i}, \varsigma_{i}\right), \\
\mathcal{U}_{0} & =\left(u_{i}^{0}, U_{i}^{0}, \varphi_{i j}^{0}, \Phi_{i j}^{0}, v^{0}, \mu^{0}, \alpha^{0}, \beta^{0}, \zeta_{i}^{0}, \varsigma_{i}^{0}\right), \\
\mathcal{F} & =\left(\mathbf{0}, f_{i}, \mathbf{0}, \ell_{i j}, 0, \ell, 0, s, \mathbf{0}, \mathcal{M}_{i}\right) .
\end{aligned}
$$

In order to transform the mixed initial-boundary problem $\mathcal{P}$ into a Cauchy problem associated with an abstract, evolutionary equation, the matrix operator $\mathcal{L}$ is introduced, having as components the operators defined by the relations (29), with the domain of definition $D(\mathcal{L})$, represented in the following

$$
\begin{aligned}
D(\mathcal{L}) & =\left(\boldsymbol{W}_{0}^{1,2} \cap \boldsymbol{W}^{2,2}\right) \times \boldsymbol{W}_{0}^{1,2} \times\left(\boldsymbol{W}_{0}^{1,2} \cap \boldsymbol{W}^{2,2}\right) \times \boldsymbol{W}_{0}^{1,2} \times \\
& \times\left(W_{0}^{1,2} \cap W^{2,2}\right) \times W_{0}^{1,2} \times\left(W_{0}^{1,2} \cap W^{2,2}\right) \times W_{0}^{1,2} \times \\
& \times\left(\boldsymbol{W}_{0}^{1,2} \cap \boldsymbol{W}^{2,2}\right) \times \boldsymbol{W}_{0}^{1,2},
\end{aligned}
$$

the significance of the Sobolev spaces $W_{0}^{1,2}, W^{2,2}, W_{0}^{1,2}$ and $W^{2,2}$ being well known. 
Using the matrix functions introduced by the relations (30), the Cauchy problem associated with the evolutionary equation has the form

$$
\begin{array}{rlr}
\frac{d \mathcal{U}}{d t} & =\mathcal{L U}(t)+\mathcal{F}(t), \quad 0 \leq t \leq t_{0}, \\
\mathcal{U}(0) & =\mathcal{U}_{0} .
\end{array}
$$

The property of the operator $\mathcal{L}$, given by the following theorem, is indispensable in proving the result regarding the existence of the previous Cauchy problem solution (32).

Theorem 4. Given that both the symmetry relations (8) and the standard hypotheses (i)-(iii) are valid, then the operator $\mathcal{L}$ is dissipative.

Proof. The conclusion of this theorem is materialized by the inequality presented by Theorem 2 , namely

$$
<\mathcal{L U}, \mathcal{U}>\leq 0, \forall \mathcal{U} \in D(\mathcal{L}) .
$$

Using the scalar product introduced by the relation (28), the operators defined by the relations (29) and considering an arbitrary element from $D(\mathcal{L})$, denoted by $\mathcal{U}$, we deduce

$$
\begin{aligned}
& <\mathcal{L} \mathcal{U}, \mathcal{U}>=-\int_{\mathcal{D}}\left(t_{j i} U_{i, j}+m_{k i j} \Phi_{i j, k}+\lambda_{j} \mu_{, j}+r_{j} \beta_{, j}+M_{i j} \zeta_{i, j}\right) d V+ \\
& +\int_{\mathcal{D}}\left[C_{i j m n} \varepsilon_{i j} \varepsilon_{m n}^{\prime}+E_{i j m n}\left(\varepsilon_{i j} \mu_{m n}^{\prime}+\varepsilon_{i j}^{\prime} \mu_{m n}\right)+F_{i j l m n}\left(\varepsilon_{i j} \gamma_{l m n}^{\prime}+\varepsilon_{i j}^{\prime} \gamma_{l m n}\right)+\right. \\
& +B_{i j m n} \mu_{i j} \mu_{m n}^{\prime}+G_{i j l m n}\left(\mu_{i j} \gamma_{l m n}^{\prime}+\mu_{i j}^{\prime} \gamma_{l m n}\right)+A_{i j k l m n} \gamma_{i j k} \gamma_{l m n}^{\prime}+B_{i j}\left(\varepsilon_{i j} v^{\prime}+\varepsilon_{i j}^{\prime} v\right)+ \\
& +C_{i j}\left(\mu_{i j} v^{\prime}+\mu_{i j}^{\prime} v\right)+D_{i j k}\left(\gamma_{i j k} v^{\prime}+\gamma_{i j k}^{\prime} v\right)+F_{i j}\left(\zeta_{i, j} v^{\prime}+\zeta_{i, j}^{\prime} v\right)+\sigma v v^{\prime}+ \\
& +d_{i j m n}\left(\varepsilon_{i j} \zeta_{m, n}^{\prime}+\varepsilon_{i j}^{\prime} \zeta_{m, n}\right)+e_{i j m n}\left(\mu_{i j} \zeta_{m, n}^{\prime}+\mu_{i j}^{\prime} \zeta_{m, n}\right)+f_{i j k m n}\left(\gamma_{i j k} \zeta_{m, n}^{\prime}+\gamma_{i j k}^{\prime} \zeta_{m, n}\right)+ \\
& \left.+A_{i j} v_{i} v_{j}^{\prime}+H_{i j}\left(v_{i} \alpha_{, j}^{\prime}+v_{i}^{\prime} \alpha_{, j}\right)+K_{i j} \alpha_{, i} \alpha_{, j}^{\prime}+g_{i j m n} \zeta_{i, j} \zeta_{m, n}^{\prime}\right],
\end{aligned}
$$

where the first integral was obtained by applying the divergence theorem and the boundary conditions.

The second integral that appears in the previous relation (34) is a form of quadratic type in relation to the following terms $\omega=\left(u_{i}, \varphi_{i j}, v, \alpha, \zeta_{i}\right), \omega^{\prime}=\left(U_{i}, \Phi_{i j}, \mu, \beta, \varsigma_{i}\right)$, so that the integral becomes

$$
\int_{\mathcal{D}} \Psi\left(\boldsymbol{\omega}, \boldsymbol{\omega}^{\prime}\right) d V=\int_{\mathcal{D}} \Psi\left(\left(u_{i}, \varphi_{i j}, \nu, \alpha, \zeta_{i}\right),\left(U_{i}, \Phi_{i j}, \mu, \beta, \varsigma_{i}\right)\right) d V .
$$

Through the previous relation (35), from the relation (34) is obtained

$$
\begin{aligned}
<\mathcal{L U}, \mathcal{U}> & =-\int_{\mathcal{D}}\left(t_{j i} U_{i, j}+m_{k i j} \Phi_{i j, k}+\lambda_{j} \mu_{, j}+r_{j} \beta_{, j}+M_{i j} \zeta_{i, j}\right) d V+ \\
& +\int_{\mathcal{D}} \Psi\left(\left(u_{i}, \varphi_{i j}, \nu, \alpha, \zeta_{i}\right),\left(U_{i}, \Phi_{i j}, \mu, \beta, \varsigma_{i}\right)\right) d V=0,
\end{aligned}
$$

which concludes the proof of this theorem.

The following theorem deduces a property of the operator $\mathcal{L}$, essential for the existence of the solution of the Cauchy problem (32). 
Theorem 5. If the symmetry relations (8) and the standard hypotheses (i)-(iii) are fulfilled, then the operator $\mathcal{L}$ satisfies the condition of range.

Proof. We say that the condition of range is satisfied by the operator $\mathcal{L}$ if the equation

$$
\mathcal{L U}=\mathcal{U}^{\prime}
$$

has at least a root $\mathcal{U}$ in $D(\mathcal{L}), \mathcal{U}^{\prime}$ being an arbitrary, fixed element from the Hilbert space $\mathcal{H}$, represented by the relation (27).

According to the operators introduced by the previous relations (29), the following vector notations will be used:

$$
\begin{aligned}
& C^{1}=\left(C_{i}^{1}\right), \quad C^{2}=\left(C_{i}^{2}\right), \quad C^{3}=\left(C_{k i}^{3}\right), \quad C^{4}=\left(C_{k i}^{4}\right), \\
& C^{5}=\left(C_{s}^{5}\right), \quad C^{6}=\left(C_{s}^{6}\right), \quad B^{1}=\left(B_{i}^{1}\right), \quad B^{2}=\left(B_{k i}^{2}\right), \\
& D^{1}=\left(D_{i}^{1}\right), \quad D^{2}=\left(D_{k i}^{2}\right), \quad A^{1}=\left(A_{i}^{1}\right), \quad A^{2}=\left(A_{k i}^{2}\right), \\
& \boldsymbol{W}=\left(W_{s}\right), \quad \boldsymbol{T}=\left(T_{s}\right), \quad \boldsymbol{V}=\left(V_{s}\right), \quad \boldsymbol{Z}=\left(Z_{s}\right) .
\end{aligned}
$$

By the instrumentality of both the previous relations (38), as well as the operators defined by the relations (29), the system of equations will become

$$
\begin{aligned}
& U=\boldsymbol{u}^{\prime}, \\
& C^{1} u+C^{2} \varphi+B^{1} v+A^{1} \beta+D^{1} \zeta=U^{\prime}, \\
& \Phi=\varphi^{\prime}, \\
& C^{3} u+C^{4} \varphi+B^{2} v+A^{2} \beta+D^{2} \zeta=\Phi^{\prime}, \\
& u=v^{\prime} \text {, } \\
& H \boldsymbol{u}+K \boldsymbol{\varphi}+E v+G \alpha+L \beta+M \zeta+F \boldsymbol{s}=\mu^{\prime} \\
& \beta=\alpha^{\prime}, \\
& R^{1} \boldsymbol{U}+R^{2} \boldsymbol{\Phi}+P v+N \alpha+S \mu+Q \boldsymbol{s}=\beta^{\prime}, \\
& \boldsymbol{s}=\boldsymbol{\zeta}^{\prime} \\
& C^{5} \boldsymbol{u}+\boldsymbol{C}^{6} \boldsymbol{\varphi}+\boldsymbol{W} v+\boldsymbol{T} \beta+\mathrm{Z} \mu+V \boldsymbol{\zeta}=\boldsymbol{\varsigma}^{\prime} .
\end{aligned}
$$

The system represented by the relations (39) can be reformulated from the perspective of the main unknowns $(\boldsymbol{u}, \boldsymbol{\varphi}, v, \alpha, \boldsymbol{\zeta})$, the other variables being attached to the right members, having the meaning of free terms, that this system will have the form

$$
\begin{aligned}
C^{1} \boldsymbol{u}+C^{2} \boldsymbol{\varphi}+\boldsymbol{B}^{1} v+D^{1} \boldsymbol{\zeta} & =\boldsymbol{U}^{\prime}-\boldsymbol{A}^{1} \alpha^{\prime} \\
C^{3} \boldsymbol{u}+C^{4} \boldsymbol{\varphi}+\boldsymbol{B}^{2} v+D^{2} \boldsymbol{\zeta} & =\boldsymbol{\Phi}^{\prime}-\boldsymbol{A}^{2} \alpha^{\prime} \\
H \boldsymbol{u}+K \boldsymbol{\varphi}+E v+G \alpha+M \boldsymbol{\zeta} & =\mu^{\prime}-L \alpha^{\prime}-F \boldsymbol{\zeta}^{\prime} \\
P v+N \alpha & =\beta^{\prime}-R^{1} \boldsymbol{u}^{\prime}-R^{2} \boldsymbol{\varphi}^{\prime}-S v^{\prime}-Q \boldsymbol{\zeta}^{\prime} \\
C^{5} \boldsymbol{u}+C^{6} \boldsymbol{\varphi}+\boldsymbol{W} v+V \boldsymbol{\zeta} & =\boldsymbol{\zeta}^{\prime}-Z v^{\prime}+\boldsymbol{T} \alpha^{\prime} .
\end{aligned}
$$


The terms that appear in the left member of each equation that compose the previous system (40) suggest the introduction of the ordered system $(\overline{\boldsymbol{u}}, \overline{\boldsymbol{\varphi}}, \bar{v}, \bar{\alpha}, \bar{\zeta})$ in the following form

$$
\begin{aligned}
\overline{\boldsymbol{u}} & =C^{1} \boldsymbol{u}+C^{2} \boldsymbol{\varphi}+\boldsymbol{B}^{1} v+D^{1} \boldsymbol{\zeta}, \\
\overline{\boldsymbol{\varphi}} & =C^{3} \boldsymbol{u}+C^{4} \boldsymbol{\varphi}+\boldsymbol{B}^{2} v+D^{2} \boldsymbol{\zeta}, \\
\bar{v} & =H \boldsymbol{u}+K \boldsymbol{\varphi}+E v+G \alpha+M \boldsymbol{\zeta}, \\
\bar{\alpha} & =P v+N \alpha, \\
\bar{\zeta} & =C^{5} \boldsymbol{u}+C^{6} \boldsymbol{\varphi}+W v+V \boldsymbol{\zeta},
\end{aligned}
$$

the scalar product being a bounded bilinear form on $W_{0}^{1,2}$.

By calculating the following scalar product, the next equality is obtained

$$
\begin{aligned}
& <(\boldsymbol{u}, \boldsymbol{\varphi}, v, \alpha, \boldsymbol{\zeta}),(\boldsymbol{u}, \boldsymbol{\varphi}, v, \alpha, \boldsymbol{\zeta})>=\int_{\mathcal{D}}\left(C_{i j m n} \varepsilon_{i j} \varepsilon_{m n}+2 E_{i j m n} \varepsilon_{i j} \mu_{m n}+\right. \\
& +2 F_{i j l m n} \varepsilon_{i j} \gamma_{l m n}+B_{i j m n} \mu_{i j} \mu_{m n}+2 G_{i j l m n} \mu_{i j} \gamma_{l m n}+A_{i j k l m n} \gamma_{i j k} \gamma_{l m n}+2 B_{i j} \varepsilon_{i j} v+ \\
& +2 C_{i j} \mu_{i j} v+2 D_{i j k} \gamma_{i j k} v+2 F_{i j} \zeta_{i, j} v+\sigma v^{2}+2 d_{i j m n} \varepsilon_{i j} \zeta_{m, n}+2 e_{i j m n} \mu_{i j} \zeta_{m, n}+ \\
& \left.+2 f_{i j k m n} \gamma_{i j k} \zeta_{m, n}+A_{i j} v_{i} v_{j}+2 H_{i j} v_{i} \alpha_{j}^{\prime}+K_{i j} \alpha_{, i} \alpha_{, j}+g_{i j m n} \zeta_{i, j} \zeta_{m, n}\right) d V .
\end{aligned}
$$

At the same time, the functions that are found on the right side member of each equation of the system (40), namely $\boldsymbol{U}^{\prime}-A^{1} \alpha^{\prime}, \Phi^{\prime}-A^{2} \alpha^{\prime}, \mu^{\prime}-L \alpha^{\prime}-F \boldsymbol{\zeta}^{\prime}, \beta^{\prime}-R^{1} \boldsymbol{u}^{\prime}-R^{2} \boldsymbol{\varphi}^{\prime}-S v^{\prime}-Q \boldsymbol{\zeta}^{\prime}$, respectively $\boldsymbol{\zeta}^{\prime}-\mathbf{Z} v^{\prime}-\boldsymbol{T} \alpha^{\prime}$, belong to the space $W^{1,2}$.

Therefore, the Lax-Milgram Theorem can be applied, see $[41,43,47]$, thus guaranteeing the existence of the solution $\mathcal{U}=\left(u, \varphi_{i j}, v, \alpha, \zeta_{i}\right)$ of the system (40), which ensures, in fact, that the system (39) has a solution. In this way, the Theorem 5 is completely proved.

By Theorems 4 and 5, it is proved that the operator $\mathcal{L}$ meets the conditions required by the Lumer-Phillips Corollary, corresponding to the Hille-Yosida Theorem, see [41,43], thereby being possible to state the theorem presented below.

Theorem 6. If the symmetry relations (8), as well as the standard hypotheses (i)-(iii) are satisfied, then the operator $\mathcal{L}$ generates a semigroup of contractions on the space $\mathcal{H}$.

Proof. Taking into account the conclusions of Theorems 4 and 5, together with the result included in the corollary Lumer-Phillips, it follows that the operator $\mathcal{L}$ generates a semigroup of contractions on the particular space $\mathcal{H}$, represented by the relation (27).

Theorem 7. Given that both the symmetry relations (8) and the standard hypotheses (i)-(iii) are valid, the initial conditions $\mathcal{U}_{0} \in D(\mathcal{L})$ and the loads $f_{i}, \ell_{i j}, \ell, s, \mathcal{M}_{i} \in C^{1}\left([0, \infty), L^{2}\right) \cap C^{0}\left([0, \infty), W_{0}^{1,2}\right)$, then the abstract problem, represented by the relation (32), admits a unique solution

$$
\mathcal{U}(t) \in C^{1}([0, \infty), \mathcal{H})
$$

Proof. The result of Lumer-Phillips Corollary, consisting in the fact that the semigroup generated by the operator $\mathcal{L}$ admits as a unique generator, the infinitesimal generator represented by the solution $\mathcal{U}(t)$, leads to the conclusion of uniqueness of this solution. 


\subsubsection{Continuous Dependence of the Solution}

Theorem 8. Assuming that the symmetry relations (8) and the standard hypotheses (i)-(iii) are accomplished. Then the function $\mathcal{U}(t)$, which is a solution of the problem (32), is continuous depending both on the initial data and on the loads $f_{i}, \ell_{i j}, \ell$, s and $\mathcal{M}_{i}$, so that

$$
|\mathcal{U}(t)| \leq \int_{0}^{t}\left\|\left(f_{i}, \ell_{i j}, \ell, s, \mathcal{M}_{i}\right)\right\| d s+\left|\mathcal{U}_{0}\right| .
$$

Proof. The deduction of the result of this theorem can be obtained with the help of the same Lumer-Phillips Corollary.

The result of the above theorem completely characterizes the solution of the Cauchy problem (32).

\section{Conclusions}

The approach of the subject regarding the effects of microtemperature on porous micromorphic thermoelastic materials, highlights the advantages of using the theory of semigroups of operators, in the context of the increased difficulty of equations and conditions, caused by the large number of unknowns.

Our qualitative results correspond to the existence, uniqueness and continuous dependence of the solution of the mixed initial-boundary value problem and are due to the flexibility of this theory. The subject development was possible naturally, even within an increasing complexity of equations and conditions governing the thermoelasticity theory of porous micromorphic materials with microtemperatures.

Author Contributions: Writing-review and editing, L.C.-M. and M.M. All authors have read and agreed to the published version of the manuscript.

Funding: This work did not receive any funding.

Conflicts of Interest: The authors declare no conflict of interest.

\section{References}

1. Cosserat, E.; Cosserat, F. Théorie des Corps déFormables; Librairie Scientifique A; Hermann et Fils: Paris, France, 1909.

2. Green, A.E.; Rivlin, R.S. Multipolar continuum mechanics. Arch. Ration. Mech. Anal. 1964, 17, 113-147. [CrossRef]

3. Mindlin, R.D. Micro-structure in linear elasticity. Arch. Ration. Mech. Anal. 1964, 16, 57-78. [CrossRef]

4. Eringen, A.C. Simple microfluids. Int. J. Eng. Sci. 1964, 2, 205-217. [CrossRef]

5. Eringen, A.C. Mechanics of Micromorphic Continua. In Mechanics of Generalized Continua, IUTAM Symposia; Springer: Berlin/Heidelberg, Germany, 1968; pp. 18-35. [CrossRef]

6. Eringen, A.C. Balance laws of micromorphic mechanics. Int. J. Eng. Sci. 1970, 8, 819-828. [CrossRef]

7. Eringen, A.C. Theory of micromorphic materials with memory. Int. J. Eng. Sci. 1972, 10, 623-641. [CrossRef]

8. Eringen, A.C. Micromorphic Elasticity. In Microcontinuum Field Theories; Springer: New York, NY, USA, 1999; pp. 269-285. [CrossRef]

9. Twiss, R.J.; Eringen, A.C. Theory of mixtures for micromorphic materials-I: Balance laws. Int. J. Eng. Sci. 1971, 9, 1019-1044. [CrossRef]

10. Twiss, R.J.; Eringen, A.C. Theory of mixtures for micromorphic materials-II. Elastic constitutive equations. Int. J. Eng. Sci. 1972, 10, 437-465. [CrossRef]

11. Gales, C. On the Nonlinear Theory of Micromorphic Thermoelastic Solids. Math. Probl. Eng. 2010, 2010, 1-16. [CrossRef]

12. He, J.H. A generalized variational principle in micromorphic thermoelasticity. Mech. Res. Commun. 2005, 32, 93-98. [CrossRef]

13. Ieșan, D. On the micromorphic thermoelasticity. Int. J. Eng. Sci. 2002, 40, 549-567. [CrossRef] 
14. Ieșan, D.; Nappa, L. On the theory of heat for micromorphic bodies. Int. J. Eng. Sci. 2005, 43, 17-32. [CrossRef]

15. Ieșan, D. Micromorphic elastic solids with initial stresses and initial heat flux. Int. J. Eng. Sci. 2011, 49, 1350-1356. [CrossRef]

16. Lee, J.D.; Chen, Y. Material forces in micromorphic thermoelastic solids. Philos. Mag. 2005, 85, 3897-3910. [CrossRef]

17. Sharma, K.; Marin, M. Reflection and transmission of waves from imperfect boundary between two heat conducting micropolar thermoelastic solids. Analele Univ. Ovidius Constanta 2014, 22, 151-175. [CrossRef]

18. Marin, M. On existence and uniqueness in thermoelasticity of micropolar bodies. C. R. Acad Sci. Paris Ser. II B 1995, 321, 375-480.

19. Romano, G.; Barretta, R.; Diaco, M. Micromorphic continua: Non-redundant formulations. Contin. Mech. Thermodyn. 2016, 28, 1659-1670. [CrossRef]

20. Cao, W.; Yang, X.; Tian, X. Basic theorems in linear micromorphic thermoelasticity and their primary application. Acta Mech. Solida Sin. 2013, 26, 161-176. [CrossRef]

21. Goodman, M.A.; Cowin, S.C. A continuum theory for granular materials. Arch. Ration. Mech. Anal. 1972, 44, 249-266. [CrossRef]

22. Codarcea-Munteanu, L.; Marin, M. A study on the thermoelasticity of three-phase-lag dipolar materials with voids. Bound. Value Probl. 2019, 2019, 1-24. [CrossRef]

23. Abbas, I.; Marin, M. Analytical solution of thermoelastic interaction in a half-space by pulsed laser heating. Phys. E Low-Dimens. Syst. Nanostruct. 2017, 87, 254-260. [CrossRef]

24. Marin, M.; Craciun, E.M.; Pop, N. Some Results in Green-Lindsay Thermoelastity of Bodies with Dipolar Structure. Mathematics 2020, 8, 497. [CrossRef]

25. Grot, R.A. Thermodynamics of a continuum with microstructure. Int. J. Eng. Sci. 1969, 7, 801-814. [CrossRef]

26. Green, A.E.; Naghdi, P.M. On thermodynamics and the nature of the second law. Proc. R. Soc. Lond. A Math. Phys. Eng. 1977, 357, 253-270. [CrossRef]

27. Ieșan, D.; Quintanilla, R. On thermoelastic bodies with inner structure and microtemperatures. J. Math. Anal. Appl. 2009, 354, 12-23. [CrossRef]

28. Bazarra, N.; Campo, M.; Fernández, J.R. A thermoelastic problem with diffusion, microtemperatures, and microconcentrations. Acta Mech. 2008, 230, 31-48. [CrossRef]

29. Chiriță, S.; Ciarletta, M.; D'Apice, C. On the theory of thermoelasticity with microtemperatures. J. Math. Anal. Appl. 2013, 397, 349-361. [CrossRef]

30. Riaz, A.; Ellahi, R.; Bhatti, M.M.; Marin, M. Study of heat and mass transfer in the Eyring-Powell model of fluid propagating peristaltically through a rectangular compliant channel. Heat Transf. Res. 2019, 50, 1539-1560. [CrossRef]

31. Marin, M.; Codarcea, L.; Chirila, A. Qualitative results on mixed problem of micropolar bodies with microtemperatures. Appl. Appl. Math. AAM 2017, 12, 776-789.

32. Marin, M.; Chirila, A.; Codarcea-Munteanu, L. On a thermoelastic material having a dipolar structure and microtemperatures. Appl. Math. Model. 2020, 80, 827-839. [CrossRef]

33. Ieșan, D.; Quintanilla, R. On a theory of thermoelasticity with microtemperatures. J. Therm. Stress. 2000, 23, 199-215. [CrossRef]

34. Ieșan, D. On a theory of micromorphic elastic solids with microtemperatures. J. Therm. Stress. 2001, 24, 737-752. [CrossRef]

35. Abd-Elaziz, E.M.; Marin, M.; Othman, M.I.A. On the effect of Thomson and initial stress in a thermo-porous elastic solid under GN electromagnetic theory. Symmetry 2019, 11, 413. [CrossRef]

36. Scalia, A.; Svanadze, M. Potential Method in the Linear Theory of Thermoelasticity with Microtemperatures. J. Therm. Stress. 2009, 32, 1024-1042. [CrossRef]

37. Svanadze, M. Fundamental solutions in the theory of micromorphic elastic solids with microtemperatures. J. Therm. Stress. 2004, 27, 345-366. [CrossRef]

38. Goldstein, J.A. Semigroups of Linear Operators and Applications, 1st ed.; University Press: Oxford, UK, 1985.

39. Marin, M.; Baleanu, D.; Vlase, S. Effect of microtemperatures for micropolar thermoelastic bodies. Struct. Eng. Mech. 2017, 61, 381-387. [CrossRef]

40. Green, A.E.; Naghdi, P.M. A re-examination of the basic postulates of thermomechanics. Proc. R. Soc. Lond. A Math. Phys. Eng. 1991, 432, 171-194. [CrossRef] 
41. Brezis, H. Functional Analysis, Sobolev Spaces and Partial Differential Equations; Springer: New York, NY, USA, 2011. [CrossRef]

42. Adams, R.A. Sobolev Spaces. In Pure and Applied Mathematics, 1st ed.; Academic Press: New York, NY, USA, 1975; Volume 65.

43. Yosida, K. Functional Analysis, 6st ed.; Springer: New York, NY, USA, 1980.

44. Ciarlet, P.G. On Korn's inequality. Chin. Ann. Math. Ser. B 2010, 31, 607-618. [CrossRef]

45. Hlaváček, I.; Nečas, J. On inequalities of Korn's type. Arch. Ration. Mech. Anal. 1970, 36, 312-334, doi:10.1007/BF00249519. [CrossRef]

46. Wang, L. On Korn's inequality. J. Comput. Math. 2003, 21, 321-324.

47. Pazy, A. Semigroups of Linear Operators and Applications to Partial Differential Equations. In Applied Mathematical Sciences Series; Springer: New York, NY, USA, 1983; Volume 44. [CrossRef]

(C) 2020 by the authors. Licensee MDPI, Basel, Switzerland. This article is an open access article distributed under the terms and conditions of the Creative Commons Attribution (CC BY) license (http://creativecommons.org/licenses/by/4.0/). 